\title{
Fabrication of Nano-Composite Photocatalyst for Hydrogen Production from Water at Neutral pH
}

\author{
Klawa S, Kite B and Diep V Ca* \\ Chemistry Department, Shenandoah University, USA
}

*Corresponding author: Diep V Ca, Professor of Chemistry, Chemistry Department, Shenandoah University, Winchester, VA 22601, USA, Tel: 15406784330; Email: dca@su.edu

\section{Research Article}

Volume 3 Issue 4

Received Date: November 16, 2018

Published Date: December 17, 2018

DOI: $10.23880 /$ nnoa- 16000152

\section{Abstract}

We report a nano-composite photocatalyst that more efficiently produced hydrogen from water at neutral $\mathrm{pH}$, compared to the photocatalysts, Pt-tipped CdSe-CdS rods reported by Amirav and coworkers. Our nano-composite photocatalyst was fabricated by coupling Pt-tipped CdSe-CdS rods with titanium oxide nanoparticles. At pH 10, this fabricated nanocomposite photocatalyst was approximately 8 times as efficient in the production of hydrogen as the original nanorods. The nano-composite photocatalyst produced hydrogen at $\mathrm{pH} 7$ with an efficiency that was $68 \%$ greater than the original nanorods.

Keywords: Photocatalyst; Hydrogen; Production

\section{Introduction}

Amirav and coworkers [1] reported a record $100 \%$ photon-to-hydrogen conversion efficiency using Pt-tipped CdSe-CdS rods. However, this efficiency was achieved only by repeatedly adding $\mathrm{KOH}$ to the reaction to maintain the $\mathrm{pH}$ at 16. In this paper, we report a nano-composite photocatalyst that produces hydrogen from water with a high efficiency at lower pHs. The nano-composite photocatalyst consists of titanium oxide coupled with the CdS quantum rods with CdSe seeds and a platinum tip at one end. When the CdSe seed absorbs light, electrons in the valence band are promoted to the conduction band, and are able to travel through the CdS rod toward the metal tip. This creates a hole in the valence band of the CdSe seed, and a negative charge on the platinum tip. Water dissociates to a certain extent into $\mathrm{OH}^{-}$and $\mathrm{H}^{+}$. The hydroxyl anion donates an electron to the hole and becomes an $\mathrm{OH}$ radical while the proton accepts an electron from the Pt tip to become a hydrogen radical. Once two hydrogen radicals have been formed, they combine to make a hydrogen gaseous molecule. A hole scavenger such as isopropyl alcohol is used to reduce the $\mathrm{OH} \bullet$ radical back to water. In Amirav and coworkers' study, high $\mathrm{pH}$ was required perhaps to ensure a sufficient source of hydroxyl anions. In our investigation, titanium oxide nanoparticles, which are coupled with the nanorods, absorb radiation at $365 \mathrm{~nm}$ and split water into $\mathrm{OH}^{-}$and $\mathrm{H}^{+}$[2]. The generated hydroxyl groups are ready to donate electrons for the reduction of $\mathrm{H}^{+}$to hydrogen gas at the nanorod photocatalyst. As a result, hydrogen can be produced at a neutral $\mathrm{pH}$. Our data showed that at $\mathrm{pH} 10$ and neutral $\mathrm{pH}$, this fabricated nano-composite photocatalyst produced hydrogen with higher efficiency than the original nanorods reported by Amirav and coworkers. 


\section{Nanomedicine \& Nanotechnology Open Access}

\section{Materials and Methods}

\section{Materials}

All chemicals with analytical grade were purchased from Sigma-Aldrich and Thermo-Fisher Scientific. $18 \mathrm{M} \Omega$ DI water was used. Tecnai Spirit TEM was used to characterize the synthesized nanorods and nanocomposite photocatalyst.

\section{Fabrication of the Nano-Composite Photocatalyst}

CdS nanorods of approximately $20 \mathrm{~nm}$ in length with a Pt tip and CdSe seeds were synthesized based on Amirav and coworkers' method [1]. To couple titanium oxide nanoparticles with the nanorods, the titanium oxide particles were synthesized [3] in the presence of the nanorods. Titanium (IV) ethoxide is used as the precursor. The size of titanium oxide nanoparticles was controlled by the concentration of Triton X-100.

\section{Synthesis of CdSe Quantum Dots}

Trioctylphosphine oxide (TOPO), octadecylphosphonic acid (ODPA), and cadmium oxide (CdO) were mixed in a 25-mL 3-neck flask (Table 1 for amounts) [1]. This mixture was placed under argon using a Schlenk line and heated to $150^{\circ} \mathrm{C}$. When the temperature reached $150^{\circ} \mathrm{C}$, the mixture was placed under vacuum for 30 minutes to remove any traces of water. The mixture was then returned to argon and heated to $370^{\circ} \mathrm{C}$. A portion of 1.805 $\mathrm{mL}$ trioctylphosphine (TOP) was injected into the flask; as soon as the temperature recovered to $370^{\circ} \mathrm{C}$, a solution of selenium in $433 \mu \mathrm{L}$ TOP was injected. The heat was removed immediately, and a several milliliters of toluene were added when the solution was cool. The resulting dark red solution was transferred to centrifuge tubes, and methanol was added to precipitate the CdSe quantum dots out of solution. The solution was then centrifuged for 30 minutes at $4000 \mathrm{rpm}$. The supernatant was discarded. The pallet was dissolved in toluene and precipitated as previously with methanol and centrifugation. This cleaning process was repeated again to yield a bright red solution in toluene.

\begin{tabular}{|c|c|c|c|}
\hline Chemical & Trial 1 & Trial 2 & Trial 3 \\
\hline TOPO (g) & 3.0045 & 3.0234 & 3.0191 \\
\hline ODPA (mg) & 282.6 & 287.0 & 279.8 \\
\hline CdO (mg) & 60.0 & 69.0 & 60.2 \\
\hline Selenium (mg) & 66.1 & 63.9 & 61.2 \\
\hline
\end{tabular}

Table 1: Amounts of chemicals used in CdSe syntheses.

\section{Synthesis of CdSe Seeded CdS Nanorods}

One half of the CdSe product was dried by gentle evaporation and the CdSe seeds were dissolved by sonication in $1.0 \mathrm{~mL}$ TOP [1]. Sulfur (Table 2 for amount) and $1.805 \mathrm{~mL}$ TOP were then added to this solution followed by additional sonication to dissolve the sulfur.

Hexylphosphonic acid (HPA), TOPO, ODPA, and CdO (Table 2 for amounts) were mixed in a separate $25-\mathrm{mL} \mathrm{3-}$ neck flask. The mixture was heated to $120^{\circ} \mathrm{C}$ under vacuum and kept under vacuum for 30 minutes. The solution was transferred to argon and heated to $320^{\circ} \mathrm{C}$ to dissolve the $\mathrm{CdO}$. The solution was then cooled back down to $120^{\circ} \mathrm{C}$ and placed under vacuum for 2 hours to remove traces of water. The solution was returned to argon and heated to $320^{\circ} \mathrm{C}$, at which $1.805 \mathrm{~mL}$ TOP were injected into the flask. As soon as the temperature recovered to $320^{\circ} \mathrm{C}$ the solution of $\mathrm{CdSe}$ and sulfur was injected into the flask. After 15 minutes, the heat was removed. Once the solution was sufficiently cool, $4 \mathrm{~mL}$ toluene were added to the dark red solution.

The solution of seeded nanorods was cleaned by 3 repeated steps of dissolving in toluene and hexane followed by precipitation with methanol and centrifugation for 15 minutes at $4000 \mathrm{rpm}$. Finally, the rods in toluene were precipitated using isopropyl alcohol as the non-solvent followed by centrifugation at $4000 \mathrm{rpm}$ for 30 minutes. One half of this product was dissolved in $3.0 \mathrm{~mL}$ dichlorobenzene (DCB) for the next synthesis.

\begin{tabular}{|c|c|c|c|}
\hline Chemical & Trial 1 & Trial 2 & Trial 3 \\
\hline TOPO (g) & 3.3500 & 3.3255 & 3.3101 \\
\hline ODPA (g) & 1.0905 & 1.0572 & 1.0044 \\
\hline CdO (mg) & 233.9 & 222.6 & 231.2 \\
\hline HPA (mg) & 77.9 & 75.7 & 73.8 \\
\hline Sulfur (mg) & 123.5 & 125.7 & 123.4 \\
\hline
\end{tabular}

Table 2: Chemicals used in synthesis of CdSe seeded CdS nanorods.

\section{Addition of Platinum Tip}

Platinum-acetylacetonate (Table 3) was added to the DCB nanorod solution and the mixture was sonicated briefly to dissolve the Pt precursor [1]. Hexadecanediol (Table 3), $10 \mathrm{~mL}$ DCB, $0.2 \mathrm{~mL}$ oleic acid, and $0.2 \mathrm{~mL}$ oleylamine were mixed in a 25 -mL 3-neck flask. The solution in the 3-neck flask was heated under vacuum to $80^{\circ} \mathrm{C}$ for 30 minutes. The solution was switched to being under argon and heated to $200^{\circ} \mathrm{C}$ at which point the $\mathrm{Pt} /$ nanorod solution was injected. The heat was removed after 5 minutes resulting in a black solution. 


\section{Nanomedicine \& Nanotechnology Open Access}

The solution was then transferred to centrifuge tubes for cleaning. Toluene was added to the solution followed by the addition of methanol to precipitate the rods. The tubes were centrifuged at $4000 \mathrm{rpm}$ for 30 minutes and the supernatant was discarded. This cleaning procedure was repeated twice more. The final pallet dissolved in toluene gave a golden-brown solution.

\begin{tabular}{|c|c|c|c|}
\hline Chemical & Trial 1 & Trial 2 & Trial 3 \\
\hline Pt-Acetylacetonate (mg) & 13.6 & 15.5 & 13.6 \\
\hline Hexadecanediol (mg) & 49.0 & 46.7 & 43.5 \\
\hline
\end{tabular}

Table 3: Chemicals used in attaching the platinum tip.

\section{Ligand Exchange}

The solution of the Pt-tipped nanorods was precipitated with methanol and centrifuged to collect the pallet. The pallet of nanorods was placed in $10 \mathrm{~mL}$ methanol [1]. Tetramethylammonium hydroxide (TMAH) and mercaptoundecanoic acid (MUA) concentrations were adjusted to dissolve the pallet in methanol. Additional TMAH and MUA was added at intervals to keep the nanorods dis solved in methanol for three hours. Toluene was then added to precipitate the rods out of solution followed by centrifugation at $4000 \mathrm{rpm}$ for 30 minutes. The pallet was dissolved in $15 \mathrm{~mL}$ ethanol, which was divided into three 5-mL portions for further experiments.

\section{Titanium Oxide Coupled Nanorods, the Nano- Composite Photocatalyst}

The procedure for coupling the nanorods with titanium oxide was carried out at room temperature under argon. A $250-\mathrm{mL}$ flask containing $100 \mathrm{~mL}$ ethanol, $0.4 \mathrm{~mL}$ of $0.1 \mathrm{M}$ Triton $\mathrm{X}-100$, and $5 \mathrm{~mL}$ of the nanorod solution from the ligand exchange was placed under argon and stirred using a magnetic stir bar. A portion of $1.7 \mathrm{~mL}$ titanium (IV) ethoxide was injected into the flask and the solution was stirred vigorously for 5 minutes. The solution was then allowed to remain under argon without stirring for 2 hours. This solution was centrifuged for 20 minutes at $4000 \mathrm{rpm}$ to collect the particles. These particles were dissolved in ethanol to give $10-\mathrm{mL}$ of solution.

\section{Characterization of the Fabricated Photocatalysts}

The nano-composite photocatalyst (titanium oxidecoupled with the Pt tipped CdSe-CdS rods), and the rods themselves were characterized by a TEM.

\section{Hydrogen Production and Evaluation}

Hydrogen production was evaluated by its power to reduce $\mathrm{Fe}^{3+}$ to $\mathrm{Fe}^{2+}$. The formation of $\mathrm{Fe}^{2+}$ ions was determined by UV-vis spectroscopy since these ions are ready to react with o-phenanthroline, forming an intensely red-colored complex, $\mathrm{Fe}(\mathrm{o} \text {-phenanthroline })_{3}{ }^{2+}$, that had a maximum absorbance at $508 \mathrm{~nm}$ [4]. By monitoring the formation of the $\mathrm{Fe}(\mathrm{o} \text {-phenanthroline })_{3}{ }^{2+}$ complex via absorbance measurements, we were able to determine relatively how much hydrogen was produced by the nano-composite photocatalyst in comparison to the lone nanorods synthesized based on Amirav and coworkers' report. Hydrogen production and evaluation was conducted in two reactors as shown in Figure 1.

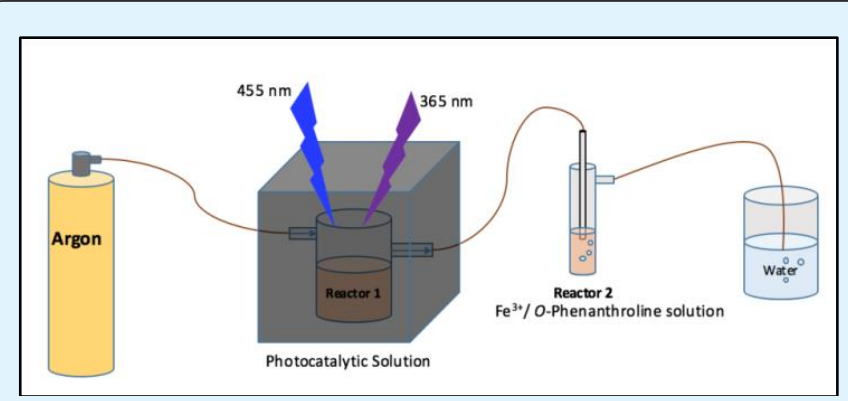

Figure 1: Diagram of reactors used to evaluate hydrogen production

The first reactor contained photocatalytic solutions, either the nano-composite photocatalyst (the titanium oxide-coupled with the Pt tipped CdSe-CdS rods) or the rods themselves. Isopropyl alcohol was added to the solution, playing a role as hole-scavenger. The solution's $\mathrm{pH}$ in the reactor 1 was adjusted by using $\mathrm{HCl}$ and $\mathrm{KOH}$ solutions. The second reactor contained $\mathrm{Fe}^{3+}$ and $\mathrm{o}^{-}$ phenanthroline solution. The reactor 1 had been illuminated for 1 hour using $365 \mathrm{~nm}$ and $455 \mathrm{~nm}$ lights while argon was flowing through the whole system. Freshly produced hydrogen from the reactor 1 was carried by argon and bubbled through the solution in the reactor 2 to reduce $\mathrm{Fe}^{3+}$ to $\mathrm{Fe}^{2+}$, which would form a complex with o-phenanthroline available in the solution. Immediately after the illumination in the reactor 1 was terminated, absorbance at $508 \mathrm{~nm}$ of the resulted solution in the reactor 2 was measured.

\section{Results and Discussion}

TEM images of the Pt tipped CdSe-CdS nanorods, and titanium oxide-coupled nanorods are shown in Figure 2. 


\section{Nanomedicine \& Nanotechnology Open Access}

From the images, the nanorods were approximately 20 nm in length.

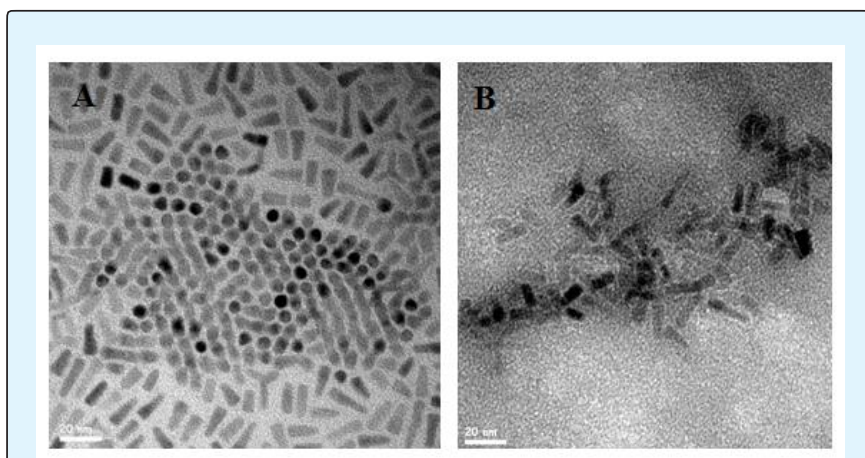

Figure 2: TEM images of (A) Pt tipped CdSe-CdS nanorods and (B) titanium oxide-coupled nanorods.

We hypothesized that the CdSe-CdS nanorods provided surface for crystallization of titanium oxide. With the concentration of the surfactant, Triton X-100, present in the mixture of titanium (IV) ethoxide precursor and CdSe-CdS nanorods solution, the TiO nanoparticles were expected to crystalize along the nanorods and form particles with size of ca. $6 \mathrm{~nm}$. However, the obtained TEM image did not seem to strongly support that hypothesis. It leads to three possibilities:

1. Titanium oxide nanoparticles indeed deposited along the CdSe-CdS nanorods. However, a higher-resolution TEM is required to reveal their sizes.

2. Titanium oxide was crystalized but as a thin layer coating the CdSe-CdS nanorods.

3. Titanium oxide nanoparticles were formed but detached and coexisted with the CdSe-CdS nanorods.

The first two possible structures of the titanium oxidecoupled nanorods are desirable because the resulting photocatlysts would be truly composite, thus would be more stable and last longer. More importantly, hydroxyl anions would be produced at close proximity to donate an electron to the CdSe seeds. In further studies, we will vary the ratio of titanium (IV) ethoxide precursor to the surfactant to achieve the desirable structure. Additional tests also need to be employed to verify structures of the resulting nano-composite photocatalysts.

Relative increase in absorbance at $508 \mathrm{~nm}$ of $\mathrm{Fe}$ (ophenanthroline) $3^{2+}$ complex in the solution in the second reactor was used to evaluate the production of hydrogen. Figure 3 shows the comparison of hydrogen produced by the titanium oxide coupled with Pt tipped CdSe-CdS nanorods, and the original nanorods at different working $\mathrm{pHs}$

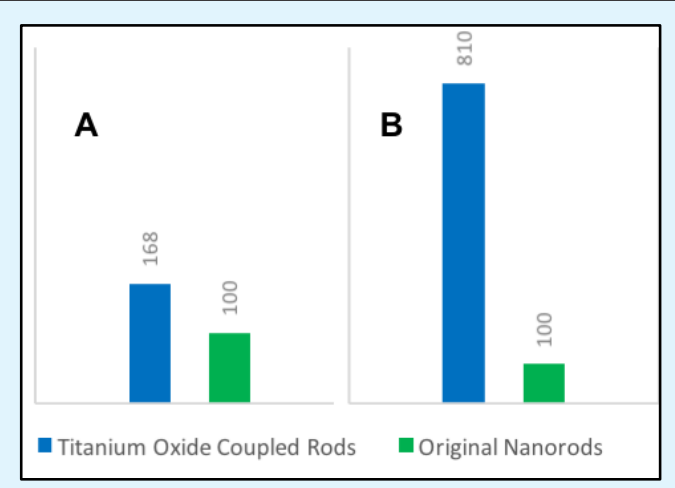

Figure 3: Relative increase in Fe (o-phenanthroline) $3_{3}^{2+}$ absorbance when photocatalysts worked at (A) $\mathrm{pH} 7$ and (B) pH 10.

As it can be seen in Figure 3, at pH 7, titanium oxidecoupled Pt tipped CdSe-CdS nanorods (nano-composite photocatalyst) produced $68 \%$ more hydrogen compared to the nanorods without titanium oxide did. This achievement proved that the present of titanium oxide is an indisputable key for the Pt tipped CdSe-CdS nanorods to reach higher production of hydrogen from water at neutral $\mathrm{pH}$. It must have been attributed to splitting water by $\mathrm{TiO}$, when being illuminated at $365 \mathrm{~nm}$, producing hydroxyl anions that were ready to donate electrons to the CdSe seeds. As a result, a greater amount of hydrogen radicals was formed at the Pt tips to produce more hydrogen gas compared to the case of absence of TiO. At $\mathrm{pH} 10$, the nano-composite photocatalyst produced approximately 8 times the amount of hydrogen as the original photocatalytic rods. This result implied that the fabricated composite nanophotocatalyst had not reached its maximum quantum yield at neutral $\mathrm{pH}$ yet, and perhaps it was due to a lack of hydroxyl anions. Therefore, an increase in the ratio of TiO-to-nanorods should be tested. In addition, effect of the TiO particles' proximity to the CdSe seeds should be examined because the TiO is responsible for the formation of hydroxyl groups that would donate electrons at CdSe site.

\section{Conclusion}

A design and fabrication of nano-composite photocatalyst, which produced hydrogen from water at neutral working $\mathrm{pH}$, is presented in this report. The nanocomposite consists of 1) $\mathrm{TiO}$ particles that split water into hydroxyl anions and protons under $365 \mathrm{~nm}$ illumination; 2) CdSe seeds that facilitate electron 


\section{Nanomedicine \& Nanotechnology Open Access}

donation of the hydroxyl anions; 3) CdS nanorod, in which CdSe is embedded, shuttle electrons to the Pt tip; 4) Pt tip (located at one end of the CdS rod), at which protons are reduced to hydrogen radicals and form hydrogen molecules. The TEM images revealed the nanocomposite photocatalyst's size of ca. $20 \mathrm{~nm}$ in length. In Amirav and coworkers, at working $\mathrm{pH}$ of 15 , the Pt tipped CdSe-CdS nanorods possessed perfect photon-to-hydrogen production efficiency. However, the requirement of extremely high $\mathrm{pH}$ conditions was the main barrier preventing this photocatalyst from practical applications. In a comparison with Amirav and coworkers' photocatalyst model, our nanocomposite photocatalyst demonstrated higher hydrogen production from water at both neutral and basic conditions. The merit of our "prove-of-concept" study enable this superior photocatalyst to work at neutral $\mathrm{pH}$, which would invite more sustainable and efficient solar-to-fuel applications. Further studies with this project aim to fine-tune the structure of the reported nanocomposite photocatalyst to maximize its quantum yield at neutral working $\mathrm{pH}$. In addition, quantitative determination of the hydrogen production will be conducted by an in-line GC-MS. A different co-photocatalyst, such as zinc oxide to split water, will be tested and compared with titanium oxide when it is coupled with the CdSe-CdS nanorods.

\section{References}

1. Kalisman P, Nakibli Y, Amirav L (2016) Perfect Photon-to-Hydrogen Conversion Efficiency. Nano Letters 16(3): 1776-1781.

2. Castellote M, Bengtsson N (2011) Application of titanium dioxide photocatalysis to construction materials. Springer: 5-10.

3. Eiden-Assmann S, Widoniak J, Maret G (2004) Synthesis and Characterization of Porous and Nonporous Monodisperse Colloidal TiO2 Particles. Chem Matter 16(1): 6-11.

4. Atkins R (1975) Colorimetric determination of iron in vitamin supplement tablets. A general chemistry experiment. J Chem Educ 52(8): 550. 